International Journal of Pure and Applied Mathematics

Volume 90 No. $1 \quad 2014,43-56$

ISSN: 1311-8080 (printed version); ISSN: 1314-3395 (on-line version)

url: http://www.ijpam.eu

doi: http://dx.doi.org/10.12732/ijpam.v90i1.6

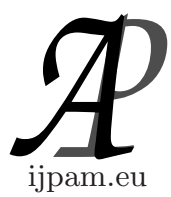

\title{
MATHEMATICAL MODELING AND BEHAVIOR OF THE DIGESTIVE SYSTEM OF A PAPER MAKING PLANT BASED ON QUEUING THEORY
}

\author{
Pervaiz Iqbal $^{1} \S$, P.S. Sehik Uduman ${ }^{2}$ \\ ${ }^{1,2}$ Department of Mathematics \\ B.S. Abdur Rahman University \\ Chennai, INDIA
}

\begin{abstract}
This paper deals with the behavior of the digestive system of a paper making plant based on queuing theory. Several methods are used to describe the behavior of some parts of the digestive system of paper making plant based on linear and non linear circuit. The digestive system of a paper making plant has four main sub-systems that are arranged in series and parallel. The mathematical local balance equations are developed using Markov Sate diagram and then solved it by using normalizing condition. The behavior of each part of the digestive system in a paper making plant has also been deduced, SLAM - II can also be used to simulate the model. Therefore, the finding of this work will be highly useful to the paper plant management for the timely execution of proper maintenance decision and hence to enhance the system performance.
\end{abstract}

AMS Subject Classification: 90Bxx, 62P30

Key Words: mathematical modeling, behavior of each part, normalizing condition, performance analysis, digestive system and queuing theory

Received: September 20, 2013

(c) 2014 Academic Publications, Ltd. url: www.acadpubl.eu

${ }^{\S}$ Correspondence author 


\section{Introduction}

The paper making industry comprises of a large complex systems that are arranged in series, parallel or a combination of both the configurations. Some of these systems are chipping, cooking, washing and bleaching, screening, stock preparation and paper making. The quality of paper mostly depends on the paper making process which is the most important process of a paper making industry. In the formation of paper making process, the chips from storage are fed into a digester to form the pulp, which is processed through various sub-systems called screw feeder, belt conveyor, shuttle conveyor and digester, the flow chart is shown in Figure 1.

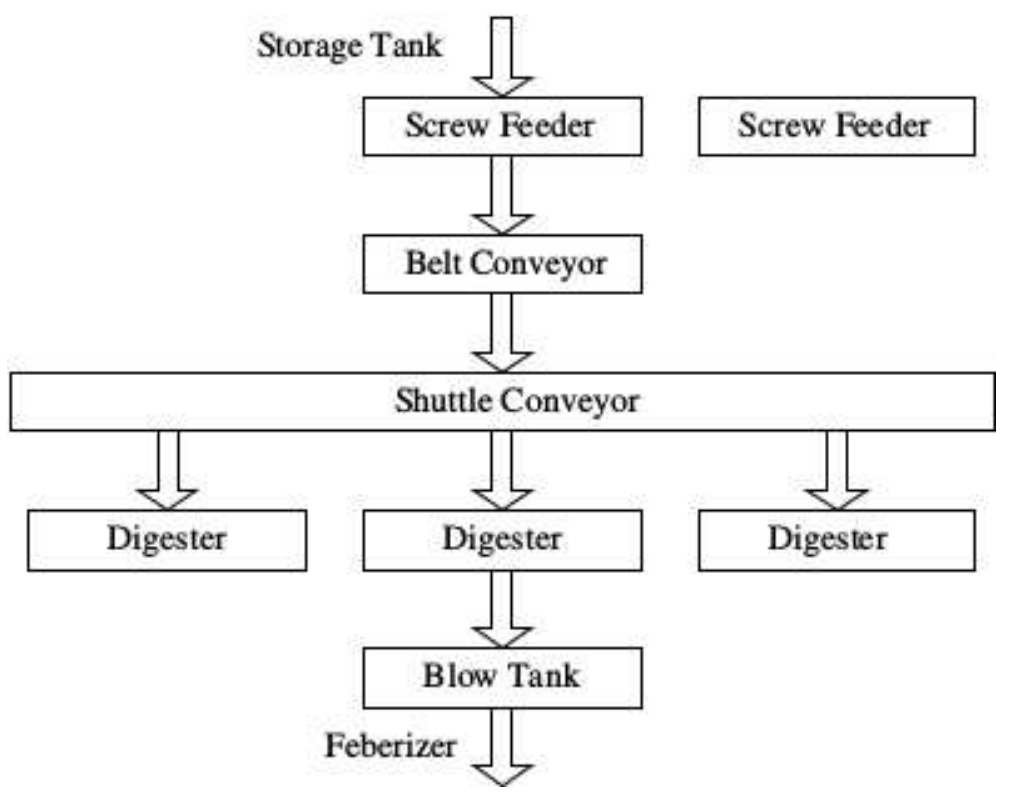

Figure 1: Schematic diagram of the digestive system of paper making plant

Paper making is the process whereby pulp fibres are mechanically and chemically treated, formed into a dilute suspension, spread over a mesh surface, the water removed by suction, and the resulting pad of cellulose fibres pressed and dried to form paper. The mechanical treatment of the fibre normally takes place by passing it between moving steel bars which are attached to revolving metal discs, the so called refiners. This treatment has two effects: it shortens the fibre (fibre cutting) and it fibrillates the fibre. The latter action increases 
the surface area, and as the fibres bond together in the paper sheet by hydrogen bonding, the increased surface area greatly increases the bonding and strength of the paper. Paper strength is dependent on the individual fibre strength and the strength of the bonds between the fibres. It is usually the latter, which is the limiting factor. Refining increases the inter-fibre bonding at the expense of the individual fibre strength, but the net result will be an increase in paper strength. Pressing and calendaring (feeding through rollers) increase density and promote smoothness. Various chemicals are added, e.g. to give water resistance, to give increased strength (see industrial resins article), to produce coloured paper, or to serve as inorganic filters.

Henry and Sealy Fourdrinier have given their name to the Fourdrinier paper machine, the first of which was first used in 1804. The stock is diluted to 0.5$1.0 \%$ consistency, and then flows as a flat stream onto an endless travelling wire screen. Water (containing a considerable amount of filter and fine material) is extracted through the wire screen, and this is then circulated back to dilute the oncoming stock. The wire and the web pass over suction boxes, and the web is finally removed from the wire at about $20 \%$ solids. After being pressed to some $35-40 \%$ solids, the web passes round a large number of steam heated cylinders to be dried until it finally contains some $95 \%$ solids and $5 \%$ water. Modern developments have tended towards so-called twin-wire machines, which overcomes the difference in two-sidedness caused by water draining through the bottom side of the web only. The twin wires are nowadays usually synthetic fibre fabrics, between which the stock is introduced.

In this paper, the mathematical (availability) model has been develop to evaluate the behavior of the digestive system of a paper making plant based on the queuing model and on the basis of certain assumptions. After that the performance optimization is done, which gives the optimum system availability levels for different combinations of arrival and processing rates of the systems of the digestive system for improving the performance of the paper making plant. So, the findings of this paper will be highly useful to the plant management in maintenance planning and control to enhance the system performance in future.

\subsection{Literature Review}

The available literature reflects that several approaches have been used to analyze the digestive system of paper making plant of various systems. Kumar et al. $[1,2]$ dealt with the maintenance planning for the system in fertilizer and thermal plants. Kumar et al. [3] dealt with reliability, availability and operational behavior analysis for different systems in paper making plant. The reliability 
of the system is determined by forming the differential equations with the help of transition diagram using Markovian approach and then solving these differential equations with the help of fourth order Runge-Kutta method. Srinath [4] has proposed the mathematical models for the prediction of reliability and availability. Sunand et al. [5] deals with the maintenance management for Ammonia Synthesis System in fertilizer plant. Fricks and Trivedi [6] stressed upon importance analysis with Markov Chains. Sunand et al. [7] discussed simulated availability of $\mathrm{CO}_{2}$ cooling system in a fertilizer plant. Barabady [8] presented a case study describing the reliability and availability analysis of crushing plant in Jajarm Bauxite mine of Iran. Rajiv et al. [9, 10] have developed performance evaluation system for screening unit of paper plants he also dealt with availability of bleaching system of paper plant. Sachdeva et al. [11] dealt with the reliability analysis of pulping system in paper industry. Rajiv also has developed decision support system for stock preparation system of paper plant. Deb [12] has explained the optimization techniques and how they can be used in the engineering problems. Tewari et al. [13,14] dealt with development of decision support system of refining system of sugar plant. Castro and Cavalca [15] presented an availability optimization problem of an engineering system assembled in series configuration which has the redundancy of units and teams of maintenance as optimization parameters. Chales and Kondo [16] tackled a multi objective combinatorial optimization problem. They used Genetic algorithm to optimize the availability and cost of a series and parallel repairable system. Pervaiz et al. [17] job sequencing problem using advanced heuristics techniques in which row sum method is discussed for solving job sequencing problem in order to minimize the total elapsed time of the sequence.

In the recent literatures it is clear that these processes are exponential processes [18]. Therefore, in our proposed approach we assume the rate of arrival of the material is Markovian and each part of the digestive system is modeled as $\mathrm{M} / \mathrm{M} / 1 / \mathrm{N}$ queue. The whole digestive system of paper making plant is modeled by a four cascaded $M / M / 1 / N$ queues which are chipping, cooking, bleaching and screening, each has its own capacity and its own serving rate. Measure this model can tell the optimum amount of material is needed for each case and can easily detect its inabilities in the queues [19]. This approach is direct and straight forward. The simulation of this system can be done by using SLAM - II [20]. 


\subsection{System Description}

The paper making system comprises of four main sub-systems, with following description:

1. Sub-system $B_{1}$ : It consists of screw feeder unit. The function of screw feeder is to extract the wooden chips from storage silos and transfer into belt conveyor.

2. Sub-system $B_{2}$ : It consists of belt conveyor unit to carry the chips.

3. Sub-system $B_{3}$ : It consists of shuttle conveyor unit to feed the wooden chips from belt conveyor to digester.

4. Sub-system $B_{4}$ : It consists of three digester units in parallel to cook the wooden chips.

\section{Analytical Modeling of the Digestive System}

The following steps for modeling of the digestive system of a paper plant:

(a) Drawing Markov state diagram

(b) Deduce the local balance equations

(c) Deduce the general recurrence equations

(d) Solving the recurrence equations and finding the values of each state probability

(e) Calculating the parameters of each part of the digestive system.

- Idle time

- Length of queue in each part

- Waiting time of certain amount of material in each part.

Assume that the capacity of each part of the digestive system of a paper plant is:

(a) The capacity of the chipping is $N_{1}$ 
(b) The permissible capacity in average for the cooking is to be filled by material is $N_{2}$ which is equal to $20 N_{1}$.

(c) The average capacity of the bleaching system is $N_{3}$ which is equal to 30 $N_{1}$.

(d) The permissible capacity in average for the screening to be filled by remainder is $N_{4}$ which is equal to $30 N_{1}$.

The block diagram of the digestive system of a paper plant is shown in Figure 2.

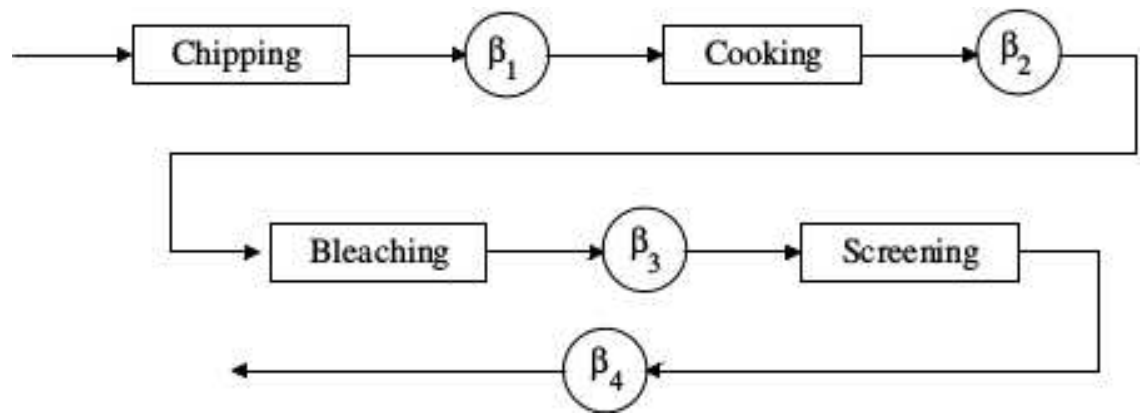

Figure 2: Block Diagram of the Digestive System of Paper Plant

\subsection{Formulation of the $M / M / 1 / N$ Queue}

Let us assume that the arriving rate for the queue is $\alpha$, the serving rate is $\beta$, the capacity of the queue is $N$, and $P_{i}$ is states of the queue where $i=0,1,2, \ldots, n$. The block diagram of this queue is given in Figure 3 .

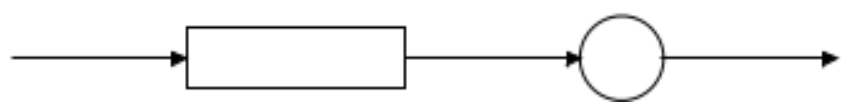

Figure 3: Block Diagram for the $\mathrm{M} / \mathrm{M} / 1 / \mathrm{N}$ Queue

The local balance equations of the queue can be deduced as:

For $n=0$ :

$$
\beta P_{1}-\alpha P_{0}=0
$$

For $n=1,2, \ldots, N-1$ :

$$
-(\alpha+\beta) P_{N}+\alpha P_{N-1}+\beta P_{N+1}=0
$$


For $\mathrm{n}=\mathrm{N}$ :

$$
-\beta P_{N}+\alpha P_{N-1}=0
$$

Let

$$
\phi=\frac{\alpha}{\beta},
$$

where $\phi$ is called the utilization factor.

By solving equation (1), (2), (3) and considering equation (4) we get:

$$
P_{n}=\phi^{n} P_{0} \text { for } 1 \leq n \leq N
$$

But using the normalizing condition i.e., sum of all probabilities is equal to one

$$
\sum_{n=0}^{N} P_{n}=1
$$

From equation (5) and (6), we can conclude

$$
P_{0}=\frac{1-\phi}{1-\phi^{N+1}}
$$

From equation (5) and (7), $P_{n}$ can be calculated as $n=0,1,2, \ldots, N$ as

$$
P_{n}=\frac{\phi^{n}(1-\phi)}{1-\phi^{N+1}}
$$

The average length of the system $L$ is:

$$
L=L_{s}+L_{q}
$$

Where $L_{s}$ is the expected length of the serving queue and $L_{q}$ is the expected length of the queue.

Also, $L$ can be calculated as

$$
L=\sum_{n \geq 0}^{N} n P_{n}
$$

Substituting equation (5) into equation (10), one can get

$$
L=\frac{1-\phi}{1-\phi^{N+1}}-\sum_{n \geq 0}^{N} n \phi^{n}
$$


Solving equation (5), we can get

$$
L=\frac{\phi\left[1+N \phi^{N+1}-(N+1) \phi^{n}\right]}{(1-\phi)\left(1-\phi^{N+1}\right)}
$$

Also, $L_{s}$ and $L_{q}$ can be calculated as given in the following equations

$$
\begin{aligned}
& L_{s}=1-P_{0} \\
& L_{q}=L-L_{e}
\end{aligned}
$$

By substituting equation (7) into equation (13), then

$$
L_{s}=\frac{\phi\left(1-\phi^{N}\right)}{1-\phi^{N+1}}
$$

By substituting equation (11) and (15) into equation (14), then

$$
L_{q}=\frac{\phi^{2}\left[1+(N-1) \phi^{N}-N \phi^{N-1}\right]}{(1-\phi)\left(1-\phi^{N+1}\right)}
$$

The mean number of material arrival and enter the digestive system per unit time is called $\alpha$ effective $\alpha_{E}$ which is equal to

$$
\alpha_{E}=\left(1-P_{N}\right)
$$

Using little's theorem we can calculate the average waiting time $W_{q}$

$$
W_{q}=\frac{L_{q}}{\alpha_{E}}
$$

\section{Formulas of the Digestive System of Paper Making Plant Using $\mathrm{M} / \mathrm{M} / 1 / \mathrm{N}$ Queues}

Let us assume that the operation in all parts of the digestive system of paper making plant is exponential distribution. Therefore, the equation (1) to (18) can be applied to study the behavior of the digestive system of a paper making plant can be summarized as following:

- The utilization factor for the chipping is

$$
\Phi_{1}=\frac{\alpha_{1}}{\beta_{1}}
$$


- The utilization factor for the cooking is

$$
\Phi_{2}=\frac{\alpha_{1}}{\beta_{2}}
$$

- The utilization factor for the bleaching is

$$
\Phi_{3}=\frac{\alpha_{1}}{\beta_{3}}
$$

- The utilization factor for the screening is

$$
\Phi_{4}=\frac{\alpha_{1}}{\beta_{4}}
$$

The idle probability for each part can be calculated by substituting equations (19) to (22) into equation (7). Also, the probability to be at any state for any part of the digestive system of paper plant can be deduced from equation (8).

Assume the operation of each part is independent. Therefore, the idle probability of all digestive systems can be expressed as:

$$
P_{01,02,03,04}=P_{01} P_{02} P_{03} P_{04}
$$

and the probability to be any state is:

$$
P_{n 1, n 2, n 3, n 4}=P_{n 1} P_{n 2} P_{n 3} P_{n 4}
$$

\subsection{Performance Analysis}

The maintenance and detailed discussion with the paper plant management, we conclude that appropriate arriving and serving rates of all the systems are selected. Then the performance analysis are prepared accordingly by putting these arriving and serving rates values form the above four different expression of utilization factor. This deals with the qualitative analysis of the factors, e.g., courses of action and state of actions, which influence the maintenance decision associated with the plant. These values are developed under the real decision making environment i.e., decision making under risk model and used to implement the proper maintenance decisions for the paper plant. 
Table 1: Availability matrices of the subsystem of paper plant

\begin{tabular}{|c|c|c|c|c|c|}
\hline \multicolumn{6}{|c|}{ Performance analysis of the subsystem of Chipping Unit } \\
\hline$\alpha_{1}$ & 0.05 & 0.10 & 0.15 & 0.20 & 0.25 \\
\hline 0.01 & 0.20 & 0.10 & 0.07 & 0.05 & 0.04 \\
\hline 0.03 & 0.60 & 0.30 & 0.20 & 0.15 & 0.12 \\
\hline 0.05 & 1.00 & 0.50 & 0.33 & 0.25 & 0.20 \\
\hline 0.07 & 1.40 & 0.70 & 0.47 & 0.35 & 0.28 \\
\hline 0.09 & 1.80 & 0.90 & 0.60 & 0.45 & 0.36 \\
\hline \multicolumn{6}{|c|}{ Performance analysis of the subsystem of Cooking Unit } \\
\hline$\alpha_{\alpha_{1}} \beta$ & 0.10 & 0.20 & 0.30 & 0.40 & 0.50 \\
\hline 0.02 & 0.20 & 0.10 & 0.07 & 0.05 & 0.04 \\
\hline 0.04 & 0.40 & 0.20 & 0.13 & 0.10 & 0.08 \\
\hline 0.06 & 0.60 & 0.30 & 0.20 & 0.15 & 0.12 \\
\hline 0.08 & 0.80 & 0.40 & 0.27 & 0.20 & 0.16 \\
\hline 0.10 & 1.00 & 0.50 & 0.33 & 0.25 & 0.20 \\
\hline \multicolumn{6}{|c|}{ Performance analysis of the subsystem of Bleaching Unit } \\
\hline$\alpha_{1}$ & 0.15 & 0.30 & 0.45 & 0.60 & 0.75 \\
\hline 0.05 & 0.33 & 0.17 & 0.11 & 0.08 & 0.07 \\
\hline 0.10 & 0.67 & 0.33 & 0.20 & 0.17 & 0.13 \\
\hline 0.15 & 1.00 & 0.50 & 0.33 & 0.25 & 0.20 \\
\hline 0.20 & 1.33 & 0.67 & 0.44 & 0.33 & 0.27 \\
\hline 0.25 & 1.67 & 0.83 & 0.55 & 0.42 & 0.33 \\
\hline \multicolumn{6}{|c|}{ Performance analysis of the subsystem of Screening Unit } \\
\hline & 0.20 & 0.40 & 0.60 & 0.80 & 1.00 \\
\hline 0.10 & 0.50 & 0.25 & 0.17 & 0.13 & 0.10 \\
\hline 0.20 & 1.00 & 0.50 & 0.33 & 0.25 & 0.20 \\
\hline 0.30 & 1.50 & 0.75 & 0.50 & 0.38 & 0.30 \\
\hline 0.40 & 2.00 & 1.00 & 0.67 & 0.50 & 0.40 \\
\hline 0.50 & 2.50 & 1.25 & 0.83 & 0.63 & 0.50 \\
\hline
\end{tabular}




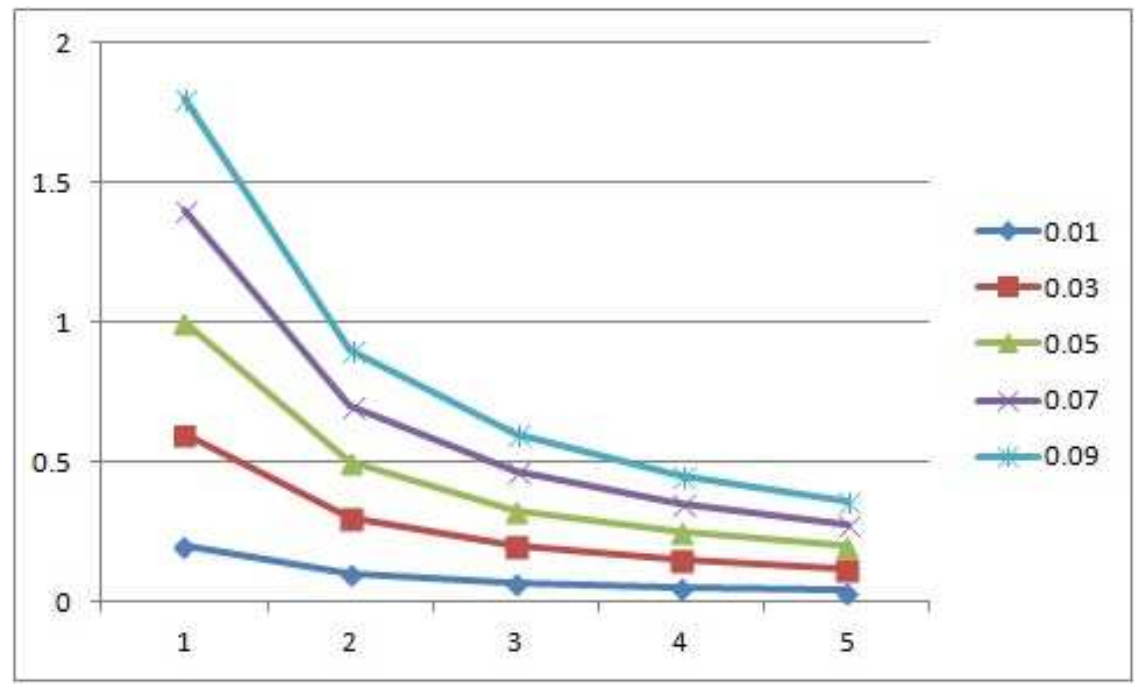

Figure 4: Performance analysis of the subsystem of Chipping Unit

Table 1 and Figure 4 shows the performance analysis for various subsystems of the paper plant. These matrices simply reveal the various performance levels for different combinations of arriving and serving rates. It also depicts the effect of arriving and serving rates parameters of all the systems on paper plant performance.

In general, when the dependent operations or for different cases the SLAM - II model will be more efficient to calculate the last parameters.

\subsection{Simulation of the Digestive System of Paper Plant IUsing SLAM - II}

The simulation of the figure first using SLAM - II nodes and symbols gives a good approach to put any new case for the cooking without need to change the algorithm or the program. This method of modeling and simulation is direct, straight forward and need less computation.

\section{Conclusion}

The mathematical modeling and behavior of the digestive system of paper plant has been carried out. The digestive system of paper plant is modeled using several queues in tandem with different serving rates. Assuming that the serving 
rate of first part is much lower than the serving rate of last and taken into consideration that the capacity of each part are different. The simulation of this model is done using SLAM - II. The best behavior of the digestive system 'chipping' is deduced. Thus the finding of this paper is discussed with the plant management. Such results are found highly beneficial for the behavior of some parts of digestive system of paper making plant.

\section{References}

[1] D. Kumar, I.P. Singh and J. Singh, Reliability analysis of the Feeding System in the Paper Industry, Microelectron Reliability, 28(2) (1988), 213215.

[2] Kumar, Dinesh, Singh, Jai and P.C. Pandey, Operational behavior and profit function for a bleaching and screening system in the paper industry, Microelectron Relaibility, 33 (1993), 1101-1105.

[3] Kumar, Dinesh, Singh, Jai and P.C. Pandey, Availability analysis of the washing system in the paper industry, Microelectron Relaibility, 29 (1989), 775-778.

[4] L.S. Srinath, Reliability Engineering, 3rd edition, East-West Press Pvt. Ltd., New Delhi, India, (1994).

[5] Sunand Kumar, Dinesh Kumar, N.P. Mehta, Maintenance management for ammonia synthesis system in a urea fertilizer plant, International Journal of Management and System (IJOMAS), 15(3) (1999), 211-214.

[6] M.F. Fricks and K.S. Trivedi, Importance analysis with Markov chains, Proceedings of the 49th Annual Reliability and Maintainability Symposium (2003), 89-95.

[7] Sunand Kumar, P.C. Tewari and Sharma Rajiv, Simulated Availability of CO2 Cooling System in a Fertilizer Plant, Industrial Engineering Journal (Indian Institution of Industrial Engineering, Mumbai), 36(10) (2007), 1923.

[8] J. Barabady and Uday Kumar, Reliability analysis of mining equipment: a case study of a crushing plant at Jajarm bauxite mine of Iran, Reliability Engineering ES System Safety, 93 (2008), 643-657. 
[9] Rajiv Khanduja, P.C. Tewari, Dinesh Kumar, Development of Performance Evaluation System for Screening Unit of Paper Plant, International Journal of Applied Engineering Research, 33 (2008), 451-460.

[10] Rajiv Khanduja, P.C. Tewari and Dinesh Kumar, Availability Analysis of Bleaching System of Paper Plant, Journal of Industrial Engineering, Udyog Pragati, N.I.T.I.E. Mumbai (India), 32(1), (2008), 24-29.

[11] Sachdeva Anish, D. Kumar and Kumar Pardeep, Reliability analysis of pulping system using Petri nets, International Journal of Quality and Reliability Management, 258 (2008), 860-877.

[12] Kalyanmoy Deb, Optimization for Engineering Design: Algorithms and Examples, Prentice Hall of India, New Delhi, India (1995).

[13] P.C. Tewari, D. Kumar, N.P. Mehta, Decision support system of refining system of sugar plant, Journal of Institution of Engineers (India), 84 (2003), 41-44.

[14] P.C. Tewari, D. Joshi, M. Sreenivasa Rao, Mathematical modeling and behavioral analysis of a refining system using Genetic Algorithm, Proceedings of National Conference on Competitive Manufacturing Technology 83 Management for Global Marketing, Chennai (2005), 131-134.

[15] H.F. Castro, K. Cavalca, Availability optimization with Genetic Algorithm, International Journal of Quality and Reliability Management, 20(7) (2003), 847-863.

[16] C. Chales, A. Kondo, Availability allocation to repairable systems with Genetic Algorithms: a multi-objective formulation, Reliability Engineering and System Safety, 82(3) (2003), 319-330.

[17] Pervaiz Iqbal, Dr. P.S. Sheik Uduman and Dr. S. Srinivasan, Job Sequencing problem Using Advanced heuristics Techniques, Proceedings of the International Conference on Applied Mathematics and Theoretical Computer Science, 1 (2013), 15-18.

[18] S.E.D. Alian, Mode Analysis and Stability for Populations of Mutually Coupled Nonlinear Oscillators, Ph. D. Thesis, Dept. of Control Eng., Sheffield University, Sheffield, England (1981).

[19] L. Kleinrock, Queueing Systclns Voliime I: Theory, John Wiley C Sons (1975). 
[20] Alan, Introduction to Simulation Language, Print Pms (1984). 